\title{
AVANÇOS E PERCALÇOS DO SUS: A REGULAÇÃO DAS RELAÇÕES ENTRE O PÚBLICO E O PRIVADO
}

\author{
ADVANCEMENTS AND DRAWBACKS OF THE UNIFIED HEALTH SYSTEM (SUS): REGULATION OF \\ THE RELATIONS BETWEEN PUBLIC AND PRIVATE SPHERES
}

Ligia Bahia ${ }^{1}$

Resumo O trabalho, elaborado como ensaio, aborda problemas decorrentes das assimetrias entre as concepções e as práticas das relações entre o público e o privado no sistema de saúde brasileiro. As hipóteses apresentadas sugerem que a preservação de arranjos político-institucionais e financeiros de suporte público aos serviços privados e empresas de planos de saúde compete com a perspectiva de implementação do direito universal à saúde. $\mathrm{O}$ argumento de que a rede privada de estabelecimentos e o empresariamento de esquemas assistenciais desoneram o SUS e, portanto, induzem ao uso eficiente dos recursos públicos para atender os que "não podem pagar"é problematizado. Considera-se, à luz de informações sobre os subsídios fiscais e gastos públicos diretos envolvidos com o financiamento dos planos privados de saúde, que a defesa de um sistema público de saúde para os pobres contrapõe-se e inviabiliza o SUS proposto pela Constituição de $1988^{2}$.

Palavras-chave SUS; planos privados de saúde; regulação.
Abstract The present essay is about problems in the Brazilian health system that originate from differences in understanding and practices of the relations between public and private spheres. The proposed hypothesis suggests that conserving political, institutional and financial arrangements for providing public support to private enterprises and HMOs is considered as important as implementing universal access to health. The article also analyses the argument that the existence of a private health sector and the financial aid given to assistance programs unburden the Unified Health System and, thus, lead to the efficient use of resources in providing care to those "who cannot pay". In light of recent information on tax-breaks and direct public expenditures destined to private HMOs, the author considers the possibility that a public health system for the poor may preclude the existence of the Unified Health System as proposed by the Brazilian Constitution of 1988.

Keyword Unified Health System; private HMOs; regulation. 


\section{Introdução}

Passados 15 anos, o SUS preconizado pela Constituição de 1988 ainda está longe de ser exeqüível. As condições atuais, desde as atinentes ao financiamento, passando pela organização institucional, até o apoio político, que caracterizam o SUS real, distanciam-no do SUS constitucional. O enfrentamento dessa disjuntiva pelas instituições setoriais evidencia profundas restrições à implantação de um sistema de saúde universal. Os diagnósticos sobre os obstáculos à consecução dos preceitos do SUS evidenciam desde problemas relacionados com a economia - submetida a severas restrições fiscais -, passando pelos relacionados com a gerência de uma unidade local, até os afeitos ao trabalho dos profissionais de saúde. Ao longo do tempo, a reiteração dessa listagem de barreiras foi sintetizada em análises aprofundadas sobre os limites e perspectivas do SUS.

A persistência do hiato entre os indicadores de saúde e o estágio de desenvolvimento econômico alcançado pelo país, bem como o descompasso entre a obtenção de direitos civis por referência aos direitos sociais - estes últimos expressos inclusive pelo acesso a cuidados e serviços de saúde de qualidade -, é atribuída à preservação da concentração de renda e à conquista de uma democracia fortemente pautada pela lógica dos interesses particularistas (quase sempre economicamente poderosos) em detrimento das normas universalistas de orientação das políticas púbicas.

A queda dos regimes autoritários em vários países da América Latinaembora indubitavelmente benéfica por seus efeitos sobre as liberdades políticas, como a ausência de coerção sobre o voto, e as liberdades de opinião e associação -, não logrou garantir direitos civis e sociais. Estabeleceu-se uma disjuntiva entre os avanços políticos e a preservação dos suportes econômico-sociais e institucionais de preservação das desigualdades sociais. Como afirmou O'Donnell (1996), os indivíduos dos países que possuem democracias informalmente institucionalizadas podem ser cidadãos em relação a uma única instituição: as eleições.

No Brasil, as grandiosas expectativas de mudanças construídas durante o processo de resistência ao regime militar e início da redemocratização embalaram a elaboração das diretrizes da Reforma Sanitária. A atmosfera de entusiasmo e esperança em relação ao futuro, compartilhada por inúmeras entidades e atores sociais, entre os quais o movimento sanitário, tinha como substrato a certeza de que a democracia acompanhar-se-ia, cedo ou tarde, por bem-estar e desenvolvimento, tal como demonstrava a experiência dos países desenvolvidos. A Constituição de 1988 é uma declaração de compromisso com a construção de um Estado democrático e de direito, cujo fundamento é a cidadania; desdobra-se na consagração do direito universal à saúde.

A desfiguração dos princípios constitucionais, especialmente os referentes ao capítulo da seguridade social, restringiu a implementação do SUS 
universal. O SUS universal - o cartão de visitas das políticas sociais no Brasil - convive com uma inequívoca estratificação do acesso aos cuidados e serviços de saúde. De fato, os 'programas universais' que não distinguem sua clientela pela capacidade de pagamento, como o da AIDS, vacinação, saúde mental e transplantes, integram a lista de êxitos de sucessivos governos e governantes. Esses programas, que, diga-se de passagem, inspiramse nos princípios da Reforma Sanitária, desafiam vários dos pressupostos contrários às políticas universalistas que hoje retornam à agenda de debates setoriais. A política de medicamentos para HIV positivos ultrapassa, na prática, as polêmicas recomendações de agências multilaterais como o FMI e o BID sobre co-pagamento, ressarcimento, subsídios fiscais para serviços e insumos de saúde. Por outro lado, a cobertura de planos privados de saúde representa a garantia de ingresso em serviços de saúde vetados para a maioria da população brasileira. O contraste entre o atendimento personalizado, com hora marcada em consultórios particulares e realização imediata de exames e procedimentos terapêuticos, e as filas e os prolongados tempos de espera para marcação de consultas e exames na rede pública confere aos planos privados de saúde o status de passaporte de saída do caos.

Esses paradoxos remetem a desafios específicos às instituições de ensino e pesquisa e aos formuladores e executores de políticas de saúde. O aprofundamento da reflexão sobre o tema 'SUS constitucional versus SUS real' requer o exame simultâneo das condições que impedem que as políticas de saúde de corte universal predominem sobre programas sociais focalizados no âmbito de um mesmo governo e, ainda, sobre os esquemas assistenciais privados. É preciso também examinar as articulações entre as políticas direcionadas a grupos vulneráveis e a privatização do sistema de proteção social, no que diz respeito especificamente à saúde. O que se constata, embora se evite afirmá-lo explicitamente, é que a agenda universalizante proposta pelo SUS constitucional tem pouca chance de ser cumprida sob as condições políticas, econômicas e institucionais que prevalecem no país.

Tais desafios impõem árduas tarefas a todos que estão envolvidos com a implementação do SUS. Para traçar um panorama mais realista e integrado a respeito do sistema de saúde brasileiro é necessário mobilizar, entre outros aportes teórico-instrumentais, acepções mais amplas sobre regulação das relações entre o público e o privado. O mapeamento dessas relações, desde aquelas essenciais - a regulação do mercado de trabalho -, é imprescindível para contextualizar o SUS como política pública e arranjo institucional dinâmico resultante de tensões, conflitos e consensos interpostos pela sociedade civil às instituições governamentais.

Visto como um conjunto de instituições, agentes e interesses, as dimensões do SUS ultrapassam em muito as limitadas fronteiras da equivocada, mas vigorosamente difundida definição do SUS como um mero convênio de 
repasse de recursos financeiros do Ministério da Saúde a entes subnacionais e destes últimos para alguns estabelecimentos e profissionais de saúde.

O resgate do SUS do confinamento administrativo dos convênios de compra e venda de serviços e das amarras do pragmatismo, que reiteram a restrição do âmbito de atuação das políticas públicas de saúde, requer a identificação das conexões entre a rede de estabelecimentos de serviços, o sistema de formação de pessoal, o setor de produção e distribuição de medicamentos, insumos e equipamentos, mídia e o exame das relações entre os poderes Executivo, Legislativo e Judiciário que modelam, sancionam, questionam e remodelam as normas formais de funcionamento do sistema de saúde. Trata-se, ainda, de desvelar as obscuras e emaranhadas regras ad hoc que dinamizam o sistema de saúde brasileiro. O modus operandi das instituições envolvidas com a saúde mantém e reproduz obstáculos ao acesso e à garantia de uma atenção personalizada, oportuna e adequada aos cidadãos brasileiros.

\section{Reconfiguração dos espaços público e privado no sistema de saúde: os planos privados}

Por seu turno, a investigação sobre as especificidades das articulações das políticas públicas com a oferta de serviços e planos privados de saúde requer a revisão dos contextos históricos de institucionalização do padrão público-privado que as constituíram e estruturaram.

Para os que consideram que o modelo de corporativismo estatal sobre o qual se erigiram as políticas previdenciárias e o Estado desenvolvimentista foi substituído pelas relações de livre mercado, a expansão dos planos privados de saúde, previdência, educação e segurança pessoal e patrimonial decorre de uma lei geral de mercado. Assim, a abertura da economia e a integração do país nos circuitos globalizados, o primado da economia sobre a política, do qual derivam as pragmáticas fórmulas de Estado e políticas sociais mínimos, desnatura a essência do SUS universal.

De outro lado, aqueles que identificam a articulação e mobilização de recursos públicos envolvidos com o suporte ao empresariamento privado da saúde opõem-se à segmentação sustentada por políticas governamentais direcionadas à proteção dos trabalhadores urbanos especializados. E de fato, a resistência de um dos sinais distintivos do corporativismo, o acesso a políticas sociais decalcadas do status sócio-ocupacional, evidencia a impropriedade das teses sobre a ruptura com o intervencionismo estatal.

Como seria possível explicar e justificar a existência de um painel de instrumentos normativos que sustentam a transferência de recursos financeiros e atribuições de assistência à saúde para os empresários, sem a parti- 
cipação do Estado? É verdade que a criação do SUS deslocou o locus de articulação dessas políticas assistenciais da previdência para a área econômica, mas não as extingüiu. A regulação das relações entre trabalhadores especializados inseridos no mercado formal de trabalho inclui os planos privados de saúde na pauta de benefícios trabalhistas e ainda faculta a dedução fiscal dos gastos com saúde para os trabalhadores formalizados e autônomos que auferem maior renda. Adicionalmente, a edição e reedição de políticas de alívio fiscal e créditos para estabelecimentos de saúde que são contratados pelas empresas de planos privados de saúde incentivam e preservam a mercantilização da atenção à saúde.

As estimativas iniciais sobre o dimensionamento dos recursos públicos envolvidos com os planos privados de saúde sugerem que o aporte público e, sobretudo, os provenientes das contribuições de empregadores e empregados repassados aos preços dos produtos e tarifas pagos por toda a sociedade seja bastante expressivo.

A elevada proporção de gastos privados com saúde, entre os quais se incluem os gastos com planos privados, tem sido apontada como fator limitante para a universalização. O fato de sermos 'americanos' na proporção de gastos privados e públicos com saúde e pretendermos construir um sistema assemelhado ao inglês tornou-se tema recorrente nos debates sobre o SUS, e está suficientemente decantado. O que parece merecer maior atenção é a explicitação das fontes, tendências e fluxos desses recursos, bem como a rede de apoios político-institucionais que os legitimam.

O documento do Conselho Nacional de Saúde intitulado "Aporte de recursos públicos para planos privados de saúde: subsídios para a apresentação do tema" (CNS, 2005) contém um conjunto sistematizado de informações a esse respeito. São identificadas três importantes fontes de recursos públicos para os planos de saúde: a dedução dos gastos com planos de saúde dos impostos devidos pelas empresas; a dedução dos gastos com planos de saúde do imposto de renda das pessoas físicas; e os gastos públicos com planos de saúde de servidores civis ${ }^{3}$. O exame da gênese e das bases de legitimação das políticas públicas de apoio aos planos privados de saúde contribui para aprofundar a reflexão sobre as relações entre o público e o privado.

Freqüentemente, o trabalhador que 'desconta para o plano' sequer sabe que sua empresa empregadora também paga parte considerável do plano de saúde (quase sempre em valores superiores aos dos empregados). As empresas de grande porte no Brasil, incluindo as estatais, gastam cerca de $10 \%$ da folha bruta de salários com o pagamento de planos de saúde para seus funcionários (Towers Perrin, 2005). A legislação que permite a essas empresas computar os gastos com planos de saúde como custo operacional, emanada pelo regime militar nos anos 60, é ainda menos conhecida, e seu mérito não foi debatido pelas instituições e entidades da saúde. O consenti- 
mento implícito sobre a repartição social dos gastos dos planos de saúde de determinados segmentos de trabalhadores é praticamente ignorado. O que os empresários dos segmentos produtivos e os especializados na comercialização de planos privados de saúde destacam em seus discursos é que estão "desonerando" o SUS.

O abatimento sem limites de gastos com assistência à saúde do Imposto de Renda de pessoa física e jurídica que redunda em renúncia fiscal e, portanto, em gasto público é um mecanismo de financiamento indireto dos planos de saúde mais divulgado. Mas não é visto com estranheza. O argumento de que os descontos com os planos de saúde serviriam para "proteger" um pouco os segmentos médios, submetidos aos notórios reajustes salariais inferiores à inflação, é amplamente disseminado e praticamente irretorquível. Nem mesmo as manifestações sobre o caráter regressivo embutido na renúncia fiscal abalam um consenso tácito sobre a inviabilidade da mudança das regras sobre o abatimento integral dos gastos com planos de saúde do Imposto de Renda.

A terceira fonte de recursos públicos para os planos privados de saúde provém dos gastos com o pagamento de planos de saúde privados para funcionários públicos. Esses gastos, que o atual Ministério do Planejamento pretende ampliar, apresentado-os como benefícios trabalhistas para garantir a cobertura de planos privados de saúde para todos os servidores federais e seus dependentes, contam com amplo respaldo de associações de funcionários e sindicatos de funcionários públicos, inclusive dos servidores das instituições de saúde.

A rarefação dos debates sobre as conexões entre o público e o privado, o desconhecimento e a fragmentação da participação das entidades sindicais nas arenas de negociação sobre a atenção à saúde reforçam as teses de "desoneração do SUS". O sentimento predominante é que "assim o SUS pode atender melhor a quem realmente não pode pagar". A acepção de que a saúde pública a ser destinada aos pobres traduz-se operacionalmente em expressões como 'SUS dependentes' e 'pagantes e não pagantes'.

Os reflexos do 'poder de pagamento' no sistema de saúde brasileiro dispensam comentários. A persistência dos diferenciais de mortalidade infantil quando dimensionada segundo classes de renda, local de moradia etc., antes atribuídos exclusivamente à pobreza, a partir da urbanização e da melhoria relativa das condições de vida passam a ser conseqüentes também às abissais diferenças no acesso e nas condições de atendimento nos serviços de saúde.

A dimensão do fenômeno que talvez mereça ser ressaltada é que o destino final da maior parcela dos recursos destinados à assistência médicohospitalar é o estabelecimento privado ou particular de saúde. Assim, os recursos públicos e gastos sociais administrados por empresas privadas e utilizados para a compra de serviços privados reiteram, ainda que sob novas roupagens, o padrão de intervenção estatal incentivado pelo regime militar. 
Como se sabe, esse ciclo de alocação e acumulação concentra recursos assistenciais nas regiões mais abastadas do país, em especial, em determinadas áreas, bairros, ruas. A ociosidade de equipamentos e capacidade instalada de determinados serviços privados e, por vezes, os preços inadequados de remuneração dessa oferta, em alguns casos excessiva e superposta - por referir-se a uma demanda relativamente restrita -, é um fator gerador de crises constantes de parte dessa rede de serviços. As conseqüências na prática de uma racionalidade que estimula a competição entre estabelecimentos semelhantes entre si pelas oportunidades oferecidas pelo mercado, contrária à da regionalização e hierarquização da rede de serviços de saúde, são sobejamente conhecidas. Contudo, as crises financeiras e as pressões dos prestadores de serviços têm sido dirimidas, quando o são, com o aporte de recursos públicos, quer sob o formato de ampliação de deduções e isenções fiscais, quer por concessão de créditos.

A adição de informações sobre a evolução dos gastos privados com planos de saúde a esse conjunto de constatações contribui para o discernimento dos espaços públicos e, portanto, dos padrões de regulação e das tendências das relações entre o público e o privado no sistema de saúde brasileiro. Segundo dados de pesquisas de orçamentos familiares (POF) do IBGE, a participação das despesas com assistência à saúde elevou-se entre 1987 e 1996 e voltou ao patamar anterior em 2003 (5,31\%, 6,5\% e 5,35\%, respectivamente). Considerando as três pesquisas, os gastos familiares com medicamentos apresentam uma tendência de crescimento (1,71\% em 1987, 1,89\% em 1996 e 2,17\% em 2003) e as demais despesas, inclusive com planos privados de saúde (0,63\% em 1987, 1,89\% em 1996 e 1,51 \% em 2003), mostram-se mais elásticas, isto é, acompanharam a queda da renda da população entre 1996 e 2003 (IBGE, 2005).

O afluxo de recursos privados no sistema de saúde é apropriado de maneira diferenciada pela rede estratificada de serviços de saúde existente no Brasil. Uma mesma cirurgia ou atendimento clínico pode ser remunerado com valores que variam em mais de 20 vezes. Esse diferencial nem sempre corresponde exatamente ao valor da mensalidade pago pelo usuário do serviço coberto por um determinado plano de saúde.

Por outro lado, os tipos de planos e, portanto, de clientelas coadunam-se com uma tipologia da demanda criada pelos próprios médicos. Os médicos 'puros' não atendem pacientes de convênios (na prática, seus clientes possuem coberturas de planos cujos valores de reembolso são suficientemente elevados). A seguir, situam-se os que atendem clientes de 'convênios bons' (na prática, os que pagam pelo menos duas vezes os valores das tabelas de remuneração). O terceiro escalão de médicos é integrado por aqueles cujos consultórios são freqüentados por clientes dos 'convênios ruins'. Restam, por fim, os 'médicos de clínica', que são os responsáveis pelos clientes que pagam diretamente preços módicos por consultas nas denominadas clínicas populares (Miranda, 2005). 
As instituições públicas de ensino e serviços de saúde desempenham um papel decisivo para a manutenção dessa estratificação. O modo de conjugação do público com o privado define os critérios para a classificação dos serviços de saúde. O médico 'puro', 'de primeira linha', está, em geral, vinculado a uma universidade pública e interna seus pacientes particulares nos hospitais privados mais caros e melhor equipados. A tentativa de trazer os pacientes particulares para os hospitais públicos - as 'duplas portas de entrada' -, encarada por muitos gestores e profissionais de saúde como única saída imediata para a crise financeira dessas instituições, a par das polêmicas sobre o conflito de interesses entre público e privado, dificulta a integração dessas instituições na rede SUS. A lógica do 'salve-se quem puder' conflita com a constituição de redes e, portanto, com a definição de perfis assistenciais complementares e não competitivos.

Esse elevado grau de imbricamento entre o público e o privado que estrutura relações financeiras e assistenciais no sistema de saúde é regulado por políticas públicas explícitas e implícitas que incluem ainda: 1) o pagamento de valores de remuneração para determinados procedimentos, por secretarias municipais e estaduais de saúde, acima da denominada tabela do SUS; 2) a contratação de cooperativas que comercializam planos de saúde para administrar serviços públicos; e 3) os sofisticados mecanismos de complementaridade da produção de ações e serviços de alto custo pelos serviços públicos, não previstos nos contratos de planos de saúde. Portanto, nesse sistema complexo, as relações de dependência e autonomia entre oferta e demanda são reguladas por políticas públicas pouco visíveis, mas efetivas.

Nesse sentido é preciso questionar as afirmações sobre a "falta de regulação" e passar a debater os conteúdos e a direcionalidade das políticas de regulação para 'desprivatizar' as relações entre financiadores, prestadores de serviços e usuários. Trata-se de centrar esforços para discriminar o público do privado, para resgatar e ampliar os espaços da saúde pública. Isso não implica a defesa da extinção das relações mercantis no sistema de saúde. Ao contrário, o que se pretende com a inflexão do trajeto dos recursos públicos em direção ao privado é que o mercado de serviços e empresas de planos de saúde não seja estendido artificialmente, mas sim, adquira dimensões compatíveis com a capacidade de financiamento privado. Isto é, mantenha-se sem os subsídios públicos que hoje lhe conferem suporte.

\section{Reflexos das relações entre o público e o privado sobre o trabalho dos profissionais de saúde}

A miríade de possibilidades de relações entre o público e o privado na assistência à saúde afeta e é reproduzida pelas múltiplas formas de inserção no trabalho dos profissionais de saúde. Os duplos e triplos vínculos dos médicos, as 
reivindicações pela diminuição informal das jornadas de trabalho do pessoal de enfermagem, e até de servidores que desempenham funções administrativas, de 40 para 30 horas e a contratação de pessoal terceirizado são reflexo e potencializam o empresariamento da saúde. A precarização dos vínculos, a informalidade das formas de recrutamento de pessoal, por vezes permeáveis ao nepotismo, e as nomeações políticas para os ocupantes de cargos técnicos nas instituições públicas tornam quase inexeqüíveis a dedicação e o compromisso dos servidores públicos com a implementação de um SUS universal.

A defesa do SUS constitucional torna-se uma tarefa de militantes, de sanitaristas, dos participantes de conselhos de saúde. Para uma parcela importante dos profissionais de saúde, o SUS significa apenas o conjunto de serviços de saúde destinado a atender os que não podem pagar. Não é por menos que esses mesmos servidores consideram imprescindível a cobertura dos planos privados de saúde. O mesmo fenômeno, examinado sob outro registro, evidencia que as demandas das entidades dos profissionais de saúde (especialmente médicas) por melhores condições de trabalho nos serviços públicos quase sempre são dissociadas das necessidades de saúde da população.

Esses limites reduzem a eficácia dos esforços de formação e capacitação de pessoal para o SUS. O investimento na implementação de modelos assistenciais baseados na integralidade não foi suficiente para contrastar a opção dos profissionais de saúde pelas especialidades requeridas pelo mercado privado de planos e serviços de saúde e o exercício de múltiplos vínculos (CFM, 2004). Segundo Almeida (2005), apesar da necessidade de formação de profissionais de novo tipo, as universidades continuam a formar os do velho tipo. Portanto, a disputa ideológica em relação à formação na graduação e na pós-graduação constitui um campo fundamental de atuação.

Outro desafio, que nem sequer consta das agendas de debate sobre o SUS, diz respeito aos conflitos de interesses entre público e privado derivados do livre trânsito de dirigentes - entre os quais secretários de saúde e diretores de unidades - de empresas privadas de saúde para as instituições públicas e vice-versa. As estreitas relações entre as Unimed's e entidades médicas são notórias. As conseqüências dessa auto-regulação ultrapassam as dimensões ético-morais. Um artigo publicado pelo Jornal do Conselho Federal de Medicina, em dezembro de 2005, revela o profundo distanciamento dos médicos dos desafios enfrentados para a implementação do SUS. Alguns médicos como Antônio Becker, autor de um texto sobre a necessidade de reajuste dos valores de remuneração dos procedimentos médico-hospitalares, consideram que "o sistema Unimed veio preencher uma lacuna aberta pela caótica saúde pública e pela desenfreada exploração das empresas mercantilistas" (CFM, 2005, p. 11). São as mesmas palavras proferidas pelo fundador da Unimed na cidade de Santos em 1967. No entanto, a repetição do argumento em um contexto totalmente distinto é potencialmente mais 
nociva, por tornar, na prática, o SUS inexistente. Tudo se passa como se fosse a mesma 'saúde pública' dos anos 60. O que existe de fato é o convênio SUS. As relações dos profissionais com as instituições públicas de saúde reduzem-se, freqüentemente, às dimensões de consumo e remuneração dos atos profissionais.

\section{Uma agenda para regular a 'desprivatização' da saúde}

Considerando que a implementação do SUS constitucional, entre outros requerimentos, depende da concentração de esforços e recursos públicos em prol da garantia do acesso universal, várias entidades, entre as quais se destaca o Conselho Nacional de Saúde, vêm elaborando uma agenda de debates e encaminhamentos sobre problemas e proposições atuais concernentes as relações entre o público e o privado na saúde. É preciso esclarecer que ainda persistem importantes divergências a respeito de alguns temas. A principal polêmica entre pesquisadores da área de saúde pública e integrantes do plenário do Conselho Nacional de Saúde refere-se aos planos para funcionários públicos. Enquanto que pesquisadores, entre os quais me incluo, consideram que a perspectiva de 'universalização' dos planos privados de saúde para funcionários públicos dever ser rechaçada, os representantes dos profissionais de saúde vêem-se pressionados por suas entidades nacionais e evitam debater o tema abertamente.

Os pontos que se seguem resultam da reflexão e de consensos estabelecidos nesses fóruns.

Somos a favor de divulgar sistematicamente o montante de recursos e 'repatriar' os recursos derivados de isenções e deduções fiscais, envolvidos com os incentivos à oferta e demanda privada, para a rede pública.

Somos contra a criação de fundos para apoiar as empresas de planos privados de saúde.

É preciso estabelecer regras para o 'trânsito' de gestores do setor privado para o público e vice-versa (critérios que explicitem e evitem o conflito de interesses).

Os planos particulares de funcionários públicos devem ser administrados por autogestões e fortalecer a rede pública de serviços de saúde.

Os critérios para priorizar o acesso aos serviços de saúde devem ser baseados exclusivamente nas condições clínicas dos pacientes e não na capacidade de pagamento (somos contra as 'duplas filas'). Esses critérios devem orientar os contratos dos estabelecimentos de saúde com o Ministério da Saúde e secretarias de saúde.

É preciso desespecializar as arenas e agendas de debate sobre o público e o privado. Compete às instâncias de debate e deliberação instituídas pelo SUS definir novas regras para a regulação entre o público e o privado na saúde. 
A instituição de instâncias de controle social da assistência suplementar é essencial. Compete ao Conselho Nacional de Saúde, em conjunto com a participação de entidades de defesa do consumidor, do Ministério Público, do Poder Judiciário, do Poder Legislativo, Ministério da Saúde e Agência Nacional de Saúde Suplementar, a formulação e o monitoramento da implementação de normas que garantam a ampliação e o uso adequado de recursos públicos para a saúde.

\section{Notas}

1 Professora do Núcleo de Estudos de Saúde Coletiva e do Laboratório de Economia Política da Saúde da Universidade Federal do Rio de Janeiro (UFRJ). Doutora em Saúde Pública pela Fundação Oswaldo Cruz (Fiocruz). <ligiabahia@terra.com.br>

2 Uma versão reduzida deste texto foi apresentada durante o Simpósio sobre Política Nacional de Saúde, Brasília, 28 e 29 de junho de 2005.

3 Os gastos públicos com a assistência de servidores militares para seus serviços próprios e os gastos de Estados e Municípios com institutos de assistência e previdência não estão sendo considerados gastos públicos com planos privados de saúde, e sim gastos públicos com esquemas assistenciais particulares. Por outro lado os gastos com planos de saúde privados de estados e municípios não foram estimados e não constam do documento do CNS.

\section{Referências}

ALMEIDA, Márcio J. 2005. Os rumos do Estado brasileiro e o SUS: recursos humanos, conquistas, desafios e dilemas. In: VII Simpósio sobre Política Nacional de Saúde, Brasília. Apresentação oral.

CNS (Conselho Nacional de Saúde). 2005. Aporte de recursos públicos para planos privados de saúde: subsídios para a apresentação do tema. Brasília. Documento para discussão.

CFM (Conselho Federal de Medicina). 2004. $O$ médico e seu trabalho: aspectos metodológicos e resultados do Brasil. Brasília: Conselho Federal de Medicina.

2005. A posição equivocada e prejudicial da Unimed. Medicina CFM, n. 157, p. 11. IBGE (Instituto Brasileiro de Geografia e Es- tatística). 2005. Pesquisa de orçamentos familiares: primeiros resultados (20022003). Rio de Janeiro: IBGE.

MIRANDA, Ana Tereza C. (2005). Valores e atitudes dos médicos e pacientes sobre o processo de decisão clínica: o caso da terapêutica hormonal no climatério. Tese de Doutorado, Rio de Janeiro: Instituto de Medicina Social, Universidade Estadual do Rio de Janeiro.

O'DONNELL, Guillermo. 1996. Uma outra institucionalização: América Latina e alhures. Lua Nova, n. 37, p. 5-56.

TOWERS PERRIN. 2005. Planos de benefícios no Brasil: $23^{a}$ pesquisa (2004). São Paulo/ Rio de Janeiro: Towers Perrin. 
